\title{
Sarcopenia como factor predictivo de dehiscencia de anastomosis en pacientes operados de cáncer de colon
}

\author{
Tomás Rodríguez C. ${ }^{1}$, Natalia Moreno B. ${ }^{1}$, Mario Abedrapo M. ${ }^{1}$, Gunther Bocic A. ${ }^{1}$, \\ Rodrigo Azolas M. ${ }^{1}$, José Luis Llanos B. ${ }^{1}$, Antonella Sanguineti M. ${ }^{1}$ y Katya Carrillo G. ${ }^{1}$
}

'Departamento de Cirugía Hospital Clínico Universidad de Chile.

Recibido el 14 de noviembre de 2018 y aceptado para publicación el 29 de enero de 2019.

Correspondencia a: Dr. Tomás Rodríguez C. dr.to.rodriguez@gmail.com
Predictive value of sarcopenia for anastomotic dehiscense in colon cancer surgery

Introduction: An important complication of colorectal surgery is anastomotic dehiscence (AD). Nutritional status is one of the important factors in $\mathrm{AD}$. An objective way to evaluate the patients' nutritional status is to measure sarcopenia, which is the reduction of skeletal muscle mass. It is possible to standardize Sarcopenia using the analysis of the Hounsfield Units (HU) and the muscular area (MA) which consider Computed Tomography of Abdomen and Pelvis (CTAP). Aim: To evaluate whether there is a relationship between $\mathrm{AD}$ and the presence of sarcopenia detected by the measurement of $\mathrm{HU}$ and MA using CTAP. The situation considers patients undergoing colectomy for cancer. Materials and Method: Cases and controls were studied with analytical statistics. 21 patients with $\mathrm{AD}$ and 40 without $\mathrm{AD}$ were chosen randomly. They include $>18$ years, with colectomy for cancer and primary anastomosis. Ostomized patients, who previous the surgery do not have CTAP or if it was not available to measure HU and MA, were excluded. The imaging evaluation was performed by an expert radiologist. Results: The comparison between groups shows that they are homogeneous with respect the sex (predominant men), age (average 60 years) and location. There are signs of imaging which suggest the presence of sarcopenia in the AD group. This is explained because there is an important statistical decrease in the $\mathrm{HU}$ values and a tendency to present lower MA values. Conclusions: The presence of sarcopenia due to alteration of $\mathrm{HU}$ in a radiological study is correlated with $\mathrm{AD}$, and could be a predictor of risk. The importance of this finding is that this risk factor is potentially correctable.

Key words: sarcopenia; colon cancer; computed topography.

\section{Resumen}

Introducción: Una complicación importante de la cirugía colorrectal es la dehiscencia de anastomosis (DA). El estado nutricional es uno de los factores importantes en la DA. Una forma objetiva para evaluar nutricionalmente a los pacientes es medir la sarcopenia, definida como disminución de masa muscular esquelética, que puede ser objetivada por análisis de Unidades Hounsfield (UH) y área muscular (AM) por medio de Tomografía Computarizada de Abdomen y Pelvis (TCAP). Objetivo: Evaluar si existe relación entre la DA y la presencia de sarcopenia detectada por medición de UH y AM en TCAP en pacientes sometidos a colectomía por cáncer. Materiales y Método: Estudio de casos y controles con estadística analítica. Se eligen de manera aleatoria 21 pacientes con DA y 40 sin DA. Se incluyen $>18$ años, con colectomía por cáncer y anastomosis primaria. Fueron excluidos pacientes ostomizados, que no tuvieran TCAP preoperatoria o que éste no permitiera medir UH y AM. La evaluación imagenológica fue realizada por radiólogo experto. Resultados: La comparación entre grupos evidencia que son homogéneos con respecto al sexo (predomino hombres), edad (promedio 60 años) y localización. Se evidencia signos imagenológicos sugerentes de sarcopenia en el grupo de DA, puesto que existe disminución en UH con valores estadísticamente significativos y tendencia a presentar valores menores en el AM. Conclusiones: La presencia de sarcopenia evaluada por alteración de UH en estudio radiológico se correlaciona con DA, pudiendo ser un predictor de riesgo. La importancia de este hallazgo es que es un factor de riesgo potencialmente corregible.

Palabras clave: sarcopenia; cáncer de colon; tomografía computada. 


\section{Introducción}

A medida que el ser humano envejece se produce una serie de cambios en los distintos sistemas del organismo, de los cuales, muchos de ellos condicionan pérdidas de funciones corporales. Uno de estos cambios es la pérdida progresiva de la masa magra junto a un incremento de la masa grasa ${ }^{1}$, fenómeno llamado sarcopenia.

Se define como sarcopenia (término derivado del griego "sarco": músculo y "penia": pérdida) a la pérdida progresiva de la masa y potencia muscular la que en general se debe, tal como se explicó inicialmente, a procesos fisiológicos del envejecimiento humano, sin embargo, se presenta también en pacientes jóvenes por otras causas como desnutrición, alteraciones endocrinas, entre otros ${ }^{2}$. Los análisis multivariados han demostrado que los principales factores de riesgo son la edad, sexo masculino y el porcentaje de grasa visceral, sin embargo, esta patología está condicionada a otros factores a considerar como déficit de vitamina $\mathrm{D}^{3,4}$, deficiencia de testosterona ${ }^{2}$, actividad proinflamatoria ${ }^{2}$, estrés oxidativo $^{5}$, disminución de ingesta proteica y porcentaje de masa grasa ${ }^{6,7}$.

Actualmente, existen métodos diagnósticos para objetivar la composición corporal y así poder evaluar objetivamente la sarcopenia como la adsorciometría dual de energía de rayos $\mathrm{X}$, el análisis de bioimpedancia eléctrica, la valoración de excreción urinaria de creatinina, antropometría, resonancia nuclear magnética y la tomografía computada (TC) ${ }^{8}$. Esta última es considerada una herramienta útil dado su disponibilidad, uso rutinario para estudio y control de pacientes oncológicos y por tener un bajo costo relativo. Distintos estudios demuestran una alta correlación entre la medición de la composición corporal total al analizar un corte axial único a nivel de la tercera a cuarta vértebra lumbar con TC evaluando el área de sección transversal de músculos psoas $^{6}$. La medición a este nivel contempla la evaluación de área muscular (AM) y del coeficiente de atenuación medido en unidades de Hounsfield (UH) el que permite evaluar densidades según contenido de grasa y músculo ${ }^{9}$.

Existen datos en la literatura que sostienen que la presencia de sarcopenia, en pacientes oncológicos, se asocia a mayor tasa de complicaciones quirúrgicas, mala respuesta a quimioterapia y a peor sobrevida $^{5}$, por lo cual es un tema a considerar a la hora de tratar a estos pacientes ${ }^{10}$.

Considerando los datos aportados por la literatura donde se evidencia la relación de pacientes sarcopénicos con peores resultados en tratamiento quirúrgico por cáncer, el objetivo de este trabajo es evaluar la relación de la sarcopenia preoperatoria pesquizada en los TC de estadificación y la dehiscencia de anastomosis en cirugía de colon por cáncer.

\section{Materiales y Método}

Se realizó estudio longitudinal retrospectivo, a través de la selección de pacientes de la base de datos oncológica del Hospital Clínico de la Universidad de Chile, operados por cáncer de colon en los años 2011 a 2017.

Fueron incluidos en la muestra aquellos pacientes que se sometieron a cirugía resectiva, con anastomosis primaria de manera electiva y con estudio de estadificación realizado en el mismo centro de salud. Todas estas cirugías fueron realizadas por cirujanos expertos, entendiendo esto a aquellos que han realizado por lo menos cincuenta procedimientos previamente.

Para la selección se excluyó a los pacientes menores de 18 años, con biopsias de pieza operatoria distinta de adenocarcinoma, pacientes sometidos a neoadyuvancia, colostomizados, antecedentes de desnutrición o cáncer previo y que no cuenten con TC preoperatorio o que este no permita la evaluación de UH ni AM.

La sarcopenia se evaluó a través de la medición del área muscular del psoas iliaco a nivel L3 y L4 y además, se realizó la medición de UH por radiólogo del centro hospitalario.

Para el análisis estadístico se utilizó STATA versión 10. Para la evaluación de frecuencias se realizó el test $\chi^{2}$ de Pearson. La normalidad de las variables se estimó con test de Shapiro-Wilks; para las diferencias de promedios se utilizó el test $\mathrm{t}$ de Student para muestras independientes y test de Levene para evaluar homocedasticidad y aquellas variables sin distribución normal se analizaron con test de Mann-Whitney. El p < 0,05 fue considerado estadísticamente significativo.

\section{Resultados}

Los pacientes seleccionados fueron 61 , de los cuales $21(34,4 \%)$ presentaron dehiscencia de la anastomosis y $40(65,6 \%)$ no presentaron dehiscencia. Todos los pacientes fueron ASA 2. El promedio general de edad de la muestra fue 64 años de los cuales $33(54 \%)$ pacientes fueron hombres. 


\section{ARTÍCULO ORIGINAL}

En el grupo de pacientes con dehiscencia de anastomosis, $12(57,1 \%)$ eran hombres, la edad promedio era de $64 \pm 12,7$ años y la ubicación del tumor más frecuente fue el colon descendente y sigmoides (47,6\%) (Figura 1).

No hubo diferencia entre ambos grupos en relación a edad, sexo, estadio tumoral (Tabla 1) y ubicación del tumor (Figura 1).
Con respecto a la medición de la sarcopenia, las áreas musculares medidas fueron menores en pacientes con dehiscencia, pero sin diferencias estadísticamente significativas (promedio del área muscular del psoas a nivel de L3: 7,16 $\pm 3,0 \mathrm{~cm}^{2}$ versus 7,73 $\pm 2,7 \mathrm{~cm}^{2}$ p 0,408 , promedio del área muscular del psoas a nivel de L4: $9,16 \pm 4,4 \mathrm{~cm}^{2}$ versus $9,5 \pm 3,2$ $\mathrm{cm}^{2} \mathrm{p} \mathrm{0,767).}$
Figura 1. Distribución de la ubicación del tumor según dehiscencia de anastomosis. Pearson $\chi^{2}=2,7665$ p 0,598.

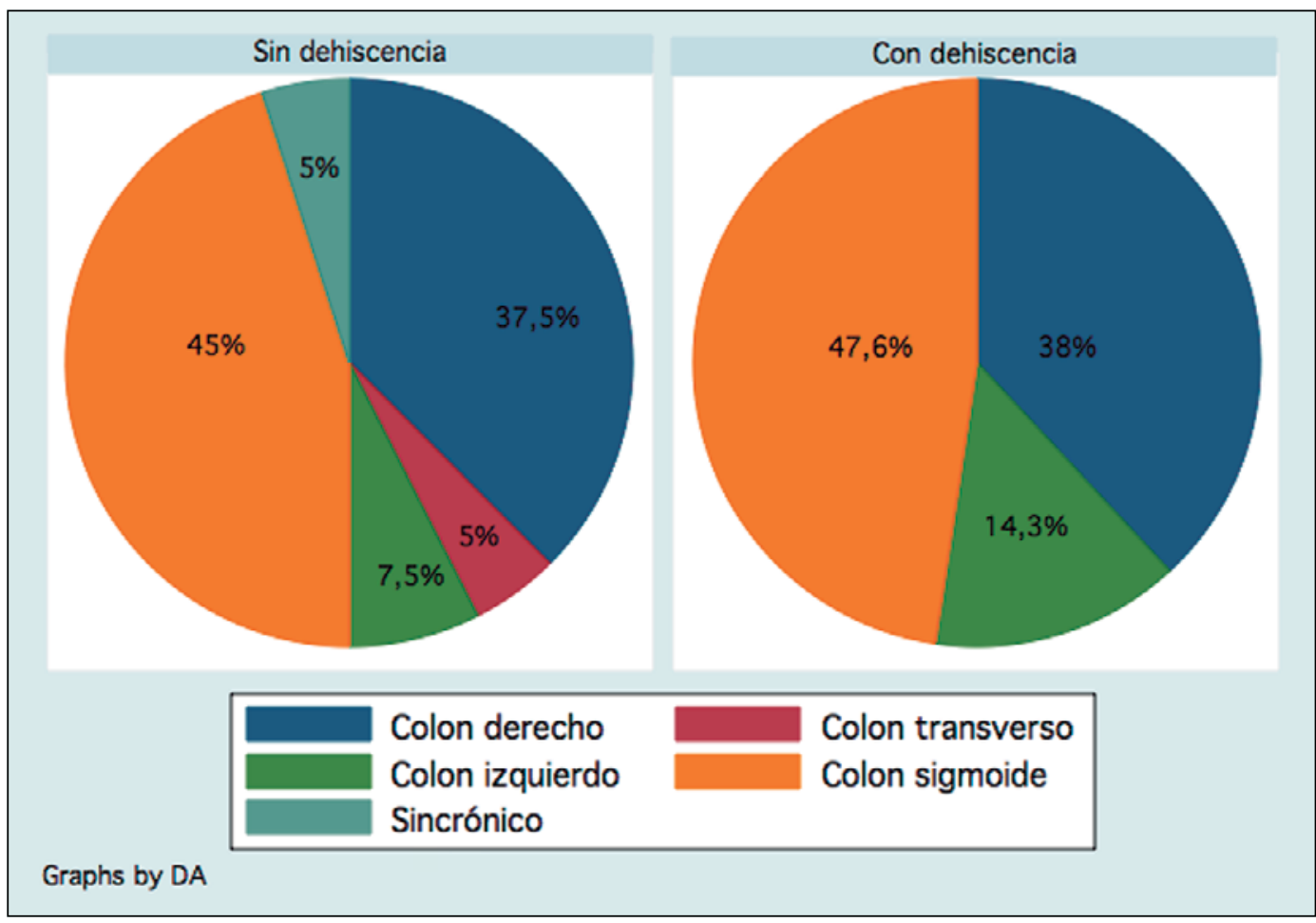

Tabla 1

\begin{tabular}{|c|c|c|c|c|c|c|c|}
\hline & & & n (\%) & Media (DS) & Total & & \\
\hline Con dehiscencia de anastomosis & $\begin{array}{l}\text { Sexo } \\
\text { Edad } \\
\text { Estadio }\end{array}$ & $\begin{array}{l}\text { Masculino } \\
\text { I } \\
\text { II } \\
\text { III } \\
\text { IV }\end{array}$ & $\begin{array}{c}12(57,1) \\
1(4,8) \\
4(19) \\
10(47,6) \\
6(28,6)\end{array}$ & $64(12,7)$ & 21 & $\begin{array}{l}\text { Pearson } \chi^{2}=0,1195 \\
\mathrm{t} \text { de Student }-0,248 \\
\text { Pearson } \chi^{2}=4,3652\end{array}$ & $\begin{array}{l}\text { p } 0,730 \\
\text { p } 0,804 \\
\text { p } 0,225\end{array}$ \\
\hline Sin dehiscencia de anastomosis & $\begin{array}{l}\text { Sexo } \\
\text { Edad } \\
\text { Estadío }\end{array}$ & $\begin{array}{l}\text { Masculino } \\
\text { I } \\
\text { II } \\
\text { III } \\
\text { IV }\end{array}$ & $\begin{array}{c}21(52,5) \\
5(12,5) \\
12(30) \\
19(47,5) \\
4(10)\end{array}$ & $63(13,8)$ & 40 & & \\
\hline
\end{tabular}


ARTíCULO ORIGINAL

Tabla 2

\begin{tabular}{|c|c|c|c|c|c|}
\hline & & UH a nivel L3 & UH a nivel L4 & Área muscular L3 & Área muscular L4 \\
\hline $\begin{array}{l}\text { Con dehiscencia } \\
\text { de anastomosis }\end{array}$ & $\begin{array}{l}\text { Media } \\
\text { Desviación estándar } \\
\text { Mínimo } \\
\text { Máximo }\end{array}$ & $\begin{array}{c}42,0000 \\
8,48086 \\
25,50 \\
57,50\end{array}$ & $\begin{array}{c}94,0952 \\
12,95726 \\
61,00 \\
115,00\end{array}$ & $\begin{array}{c}7,1690 \\
3,04871 \\
2,60 \\
13,75\end{array}$ & $\begin{array}{c}9,1619 \\
4,42049 \\
1,65 \\
17,80\end{array}$ \\
\hline $\begin{array}{l}\text { Sin dehiscencia de } \\
\text { anastomosis }\end{array}$ & $\begin{array}{l}\text { Media } \\
\text { Desviación estándar } \\
\text { Mínimo } \\
\text { Máximo }\end{array}$ & $\begin{array}{c}51,4625 \\
8,04745 \\
35,00 \\
64,50 \\
\text { Levene } 0,804 \\
\text { t de Student } \mathrm{t}=4,284 \\
\mathrm{p}<0,001\end{array}$ & $\begin{array}{c}108,5750 \\
12,15685 \\
83,00 \\
127,00 \\
\text { Levene } 0,949 \\
\text { t de Student } t=4,321 \\
\mathrm{p}<0,001\end{array}$ & $\begin{array}{c}7,7363 \\
2,74069 \\
3,90 \\
16,50 \\
\text { Mann-Whitney } \\
\text { p } 0,408\end{array}$ & $\begin{array}{c}9,5063 \\
3,26774 \\
3,75 \\
17,00 \\
\text { Mann-Whitney } \\
\text { p } 0,767\end{array}$ \\
\hline
\end{tabular}

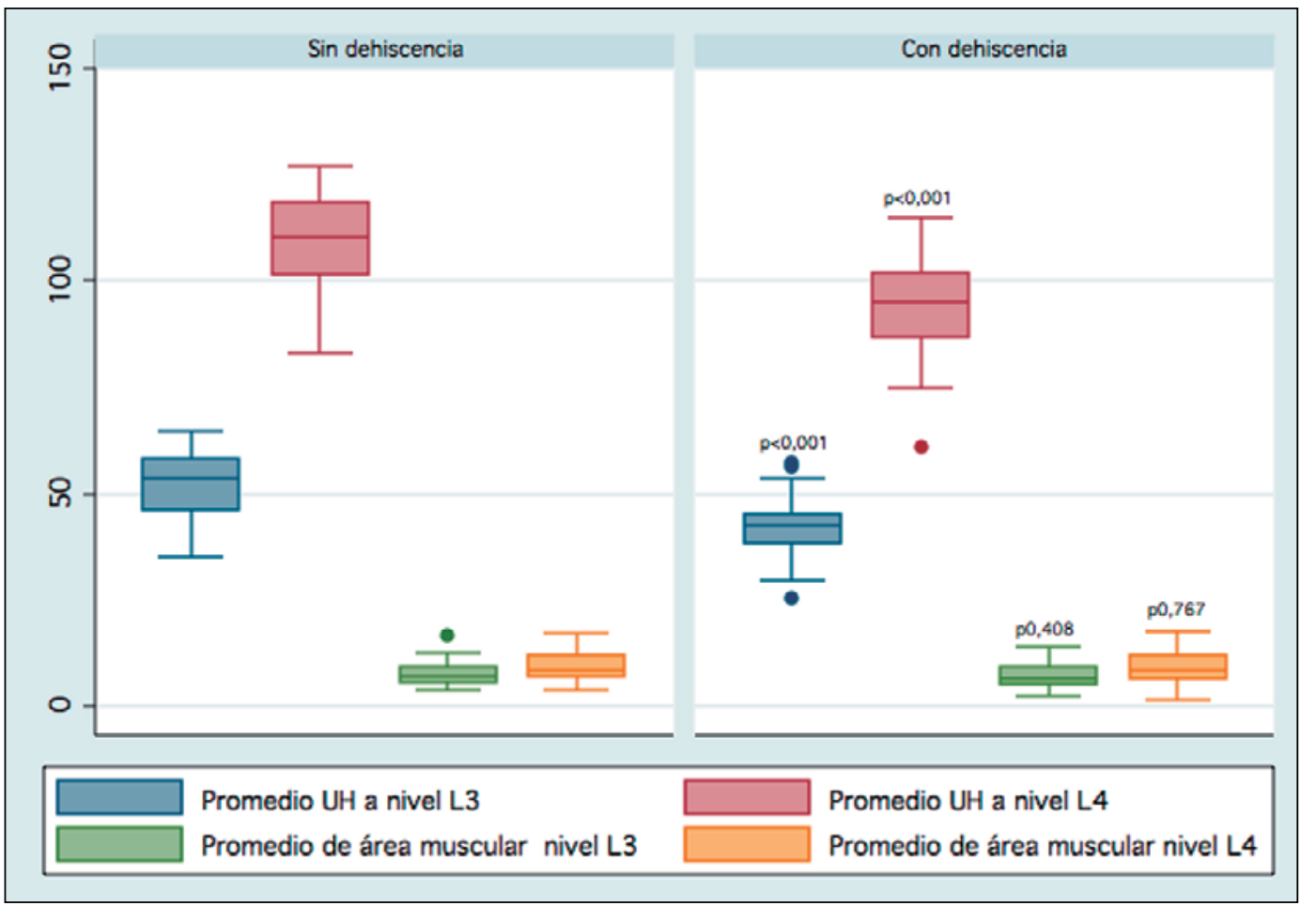

Figura 2. Medición de sarcopenia según HU y áreas musculares psoas iliaco.
Cuando se comparó la densidad, las HU promediadas también fueron menores con diferencias estadísticamente significativas (HU L3 promedio 42 $\pm 8,481$ vs $51,463 \pm 8,047 \mathrm{p}<0,0001$ y a nivel de L4 promedio $94,10 \pm 12,96$ versus $108,5 \pm 12,16 \mathrm{p}$ $<0,0001$ ) (Tabla 2 y Figura 2).

\section{Discusión}

Desde hace mucho tiempo se conoce la fuerte asociación entre cáncer colorrectal y desnutri- ción, grupo al cual pertenece la sarcopenia. Se ha estudiado ampliamente que esta asociación está relacionada directamente a peores resultados oncológicos y quirúrgicos afectando la sobrevida de estos enfermos ${ }^{2,8,10,11}$. Existen estudios donde esta asociación, mortalidad por cáncer colorrectal y sarcopenia, se duplica, si a esto se agrega inflamación sistémica ${ }^{12}$, lo cual resulta interesante ya que son aspectos potencialmente tratables, lo que determinaría un cambio en el pronóstico de estos pacientes.

También se ha identificado que la sarcopenia es 
un factor pronóstico independiente de morbimortalidad asociado a otros cánceres como páncreas, gástrico, pulmón y vía biliar ${ }^{13}$. Estudios internacionales desmuestran que la sarcopenia no solo determina peores resultados quirúrgicos en pacientes oncológicos, sino también es factor pronóstico de aumento de infecciones ${ }^{10}$, toxicidad en neoadyuvancia $\mathrm{y}$ adyuvancia $^{14}$, aumenta los días de hospitalización y alarga los planes de rehabilitación ${ }^{10}$.

La tomografía computada como método para evaluación de sarcopenia es un método aceptado y utilizado por la comunidad médica sobre todo en pacientes oncológicos. Existe literatura donde evidencia que la presencia de sarcopenia está asociada a peores resultados oncológicos y quirúrgicos ${ }^{9,10,13}$, por lo que creemos importante poder pesquisarla previo al inicio de tratamientos oncológicos para poder optimizar los resultados.

En una revisión sistemática y metaanálisis realizada por un grupo de investigadores chinos publicado el año $2018^{15}$, se presenta a la sarcopenia como un factor pronóstico para los pacientes tratados de cáncer colorrectal no metastásico en donde los resultados quirúrgicos son peores comparados con pacientes sin sarcopenia. Estos datos son similares a los obtenidos en nuestra experiencia.

Al igual que estudios nacionales e internacionales $^{9,16-18}$, en nuestro estudio se observaron peores resultados quirúrgicos evidenciando aumento de dehiscencia de anastomosis en pacientes que presentaron signos imagenológicos de sarcopenia en el TC preoperatorio, existiendo una correlación estadísticamente significativa entre la densidad evaluada (UH) y la dehiscencia de anastomosis.

\section{Conclusiones}

La densidad y área muscular traduce calidad y composición muscular, las cuales pueden ser evaluadas con distintos métodos los que ya fueron mencionados. Es importante identificar esta disminución de masa muscular en pacientes con cáncer de colon ya que este problema, la sarcopenia, eleva la morbilidad. Los pacientes con cáncer de colon son un grupo importante que deberían ser estudiados ya que la presencia de sarcopenia identificada en imágenes de TC está asociada a un aumento de complicaciones operatorias como dehiscencia de anastomosis, elevando la morbilidad y días de hospitalización independiente del sexo, edad, sitio y estadio del tumor.

Como grupo creemos que en todos los pacientes con cáncer de colon que requieran tratamiento quirúrgico, debe ser evaluada la presencia de sarcopenia para poder realizar cambios y optimizar resultados, sin embargo, es necesario realizar estudios prospectivos para unificar criterios y realizar protocolos de trabajo.

\section{Responsabilidades éticas}

Protección de personas y animales. Los autores declaran que para esta investigación no se han realizado experimentos en seres humanos ni en animales.

Confidencialidad de los datos. Los autores declaran que en este artículo no aparecen datos de pacientes.

Conflictos de interés: no hay.

\section{Bibliografía}

1. Masaés F, Navarro M, Sacanella E, López A. ¿Qué es la sarcopenia? Semin Fund Esp Reumatol. 2010;11:14-23.

2. Serra J. Consecuencias clínicas de la sarcopenia. Nutr Hosp. 2006;21:46-50.

3. Robinson S, Cooper C, Aihie Sayer A. Nutrition and sarcopenia: a review of the evidence and implications for preventive strategies. J Aging Research 2012; 2012:1-6. doi:10.1155/2012/510801

4. Sakuma K, Yamaguchi A. Sarcopenia and age-related endocrine function. Int J Endocrinol. 2012;2012:1-10. doi:10.1155/2012/127362.

5. Rom O, Kaisari S,Aizenbud D, Reznick
AZ. Lifestyle and sarcopenia-etiology, prevention, and treatment. Rambam Maimonides Med J. 2012;3:e0024.

6. Krause KE, McIntosh EI, Vallis LA. Sarcopenia and predictors of the fat free mass index in community-dwelling and assisted-living older men and women. Gait \& posture 2012;35:180-5.

7. Baumgartner RN, Koehler KM, Gallagher D, Romero L, Heymsfield SB, Ross RR, et al. Epidemiology of sarcopenia among the elderly in New Mexico. Am J Epidemiol. 1998;147:755-63.

8. Canda A. Puntos de corte de diferentes parámetros antropométricos para el diagnóstico de sarcopenia. Nutr Hosp. 2005:32;765-70.
9. Alvayay P, von Geldern O, De la Maza M, Silva C. Área muscular abdominal determinada por tomografía computada como predictor de mortalidad en pacientes oncológicos. Rev Chil Radiol. 2015;21:133-7.

10. Lieffers J, Bathe O, Fassbender K, Winget M, Baracos V. Sarcopenia is associated with postoperative infection and delayed recovery from colorrectal cáncer resection surgery. British Journal of Cancer 2012;107: 931-6.

11. Cawthon PM, Fox KM, Gandra SR, Delmonico MJ, Chiou CF, Anthony MS, et al. Health, aging and body composition study. Do muscle mass, muscle density, 
ARTÍCULO ORIGINAL

strength, and physical function similarly influence risk of hospitalization in older adults? Journal of the American Geriatrics Society 2009;57:1411-9.

12. Céspedes E, Kroenke C, Meyerhardt J. Association of Systemic Inflammation and Sarcopenia With Survival in Nonmetastatic Colorectal Cancer Results From the C SCANS Study. JAMA Oncol. 2017;3:e172319.

13. Mourtzakis M, Prado CMM, Lieffers JR, Reiman T, McCargar LJ, Baracos VE. A practical and precise approach to quantification of body composition in cancer patients using computed tomography images acquired during routine care. Appl Physiol Nutr Metab. 2008;33:997-1006.

14. Berret M, Antoun S, Dalban C, Malka D, Mansourbakht T, Zaanan A. Sarcopenia Is Linked to Treatment Toxicity in Patients With Metastatic Colorectal Cancer. Nutrition and Cancer 2014:66;583-9.

15. Sun G, Li Y, Peng Y, Lu D, Zhang F, Cui $\mathrm{X}$, et al. Can sarcopenia be a predictor of prognosis for patients with non-metastatic colorectal cancer? A systematic review and meta-analysis. Int J Colorectal Dis. 2018:33;1419-27.

16. Studley HO. Percentage of weight loss. A basic indicator of surgical risk in patients with chronic peptic ulcer. JAMA 1936;106:458-60.

17. Reisinger W, Van Vugt L, Tegels J, Snijders C, Hulsewél W. Functional Compromise Reflected by Sarcopenia, Frailty, and Nutritional DepletionPredicts Adverse Postoperative Outcome After Colorectal Cancer Surgery. Annals of Surgery 2015;261;345-52.

18. Martin L, Birdsell L, MacDonald N, Reiman T, Clandinin MT, McCargar L, et al. Cancer cachexia in the age of obesity: skeletal muscle depletion is a powerful prognostic factor, independent of body mass index. Journal of Clinical Oncology 2013;31:1539-47. 